\title{
DIRE CE QUI NE S'EST PAS PRODUIT : KANA SA-YAF 'ALU \\ (IL AURAIT FAIT ; IL ALLAIT FAIRE / HE WOULD HAVE DONE ; HE WAS GOING TO DO) \\ EN ARABE CONTEMPORAIN
}

\author{
Catherine PINON
}

IREMAM

\section{Résumé}

Dans cet article, nous proposons d'étudier en arabe contemporain la structure kāna sayaf'alu (en français « il allait faire »; en anglais " he was going to do »). Elle peut être analysée de deux manières différentes : soit il s'agit du verbe kāna à valeur syntaxique associé à une particule et dans le champ duquel entre le futur formel sa-yaf'alu, soit il s'agit d'une seule et même structure intelligible comme telle. Après avoir fait la synthèse des apparitions de kāna sa-yaf'alu dans les grammaires d'arabe moderne, nous proposons une description systématique des deux types d'emploi en inventoriant les différentes valeurs qu'elle véhicule.

Mots clés

kāna sa-yaf'al, linguistique arabe, linguistique de corpus, arabe contemporain, syntaxe, pragmatique, conditionnelles, interrogatives indirectes, concessives, contrefactualité 
1. Introduction

Dans un récent article, Pierre Larcher s'interrogeait sur une structure syntaxique originale en arabe, kāna sa-yaf'alu, que nous pouvons traduire en français par «il allait faire » et en anglais par « he was going to do » ${ }^{1}$. Après avoir exclu qu'il s'agît d'une structure propre à l'arabe moderne, il laissait entrevoir l'éventail de valeurs que peut recouvrir cette structure, qui, tout comme « il allait faire » en français, connaît des emplois allant du factuel au contrefactuel en passant par toute une gamme de nuances. Il posait, en filigrane, la question de l'origine structurelle de ces emplois : étaient-ils inspirés des dialectes ou des langues européennes ? En proposant quelques pistes, cet article laissait donc toute la voie à une enquête sur les valeurs prises en charge par kāna sa-yaf'alu afin d'éclaircir ce phénomène linguistique.

Nous nous proposons donc d'étudier ici en détail la structure kāna sa-yaf'alu dans un corpus d'arabe contemporain afin de décrire et de classer ses différents emplois. Ce classement sera pour nous l'occasion de faire le point sur les descriptions de cette structure dans les grammaires de l'arabe et de les comparer à nos résultats. La question de son origine historique sera aussi posée, et nous tenterons de faire apparaître le rôle que peuvent y jouer les dialectes arabes et les langues européennes comme l'anglais et le français.

La suite kāna sa-yaf'alu peut être analysée de deux manières différentes. Soit il s'agit du verbe kāna à valeur syntaxique associé à une particule et dans le champ duquel entre le futur formel sa-yaf'alu, soit il s'agit d'une seule et même structure intelligible comme telle. Nous décrirons les contextes d'apparition syntaxiques des deux différents types, ainsi que les valeurs qu'y portent la structure.

2. Les descriptions existantes de kāna sa-yaf'alu dans les grammaires de l'arabe ${ }^{2}$

Bien que seules des grammaires postérieures à l'an 2000 mentionnent cet emploi, il

\footnotetext{
${ }^{1}$ Larcher, Pierre, « Une occurrence ancienne de la structure kāna sa-yaf'alu en arabe écrit », à paraître.

${ }^{2}$ La liste des 15 grammaires arabes, 6 dictionnaires arabes et 55 grammaires arabisantes qui ont été consultés pour cette recherche est fournie dans Pinon (Nébuleuse, p. 469-474). Toutes les mentions de la structure que nous y avons trouvées sont citées dans le présent article.
} 
n'est pas récent puisqu'on le trouve chez Sïbawayhi ${ }^{3}$, mais probablement assez rare pour avoir échappé aux grammaires arabes. La structure kāna sa-yaf'al fait son apparition dans deux grammaires d'arabe moderne publiées en 2004 où l'on ne compte cependant qu'assez peu d'attestations.

Dans un paragraphe consacré aux conditionnelles purement contrefactuelles, Badawi, Carter et Gully ${ }^{4}$ donnent l'exemple suivant, où kāna sawfa yaf'alu se trouve dans une complétive dépendante du verbe de la protase ${ }^{5}$ :

(1) Rubbamā law 'arafa zawğuhā anna l-ḩawḍa fī tafāṣîlihā atnā’a miḥnatihā kāna sawfa yusā iduhā wa-yuqarribuhā minhu la-nșā'a li-rağbatihā. "Perhaps if the husband had known that going into details during her torment would have helped her and brought her nearer to him, [then] he would have yielded to her wish. » «Peut-être que si son mari avait su qu'entrer dans les détails lors de son épreuve l'aurait aidée et l'aurait rapprochée de lui, il aurait cédé à son désir.»

Les auteurs choisissent de traduire en anglais par un conditionnel passé. Notons au passage qu'il s'agit du seul exemple avec sawfa, peut-être pour renforcer, rhétoriquement, le caractère certain du résultat de l'action qui n'a pas été accomplie (s'il avait su, et donc avait cédé à son désir, cela l'aurait certainement aidée et elle se serait nécessairement rapprochée de lui). Cet exemple n'étant pas référencé, il n'est pas possible d'accéder à un contexte plus large afin de mieux comprendre l'occurrence.

\footnotetext{
${ }^{3}$ Sībawayhi, Al-Kitāb, éd. Hārūn 'Abd as-Salām, Le Caire, Al-hay'a l-mișriyya l- 'āmma li-l-kitāb, 5 volumes, 1977. Cf. vol. IV, p. 224 : «wa-ammā "law" fa-li-mā kāna sa-yaqa 'u li-wuqū' ġayri-hi ». Cet exemple est repris par Kees Versteegh, « Two conceptions of irreality in Arabic grammar : Ibn Hišām and Ibn al-Hāăib on the particle law », dans Pierre Larcher, De la grammaire de l'arabe aux grammaires des arabes, BEO, XLIII, Damas, IFEAD, 1991, p. 77-91. K. Versteegh semble être le premier à avoir cité cette définition, reprise par Giolfo, Manuela, Les systèmes hypothétiques en 'in de l'arabe classique: étude syntaxique et sémantique : une hypothèse modale, Aix-enProvence, thèse de doctorat inédite, 2010 et enfin Larcher, Occurrence. On voit bien que l'auteur utilise luimême cette structure dans ses explications, mais qu'elle n'est pas l'objet de sa description. Il est d'ailleurs intéressant de noter qu'il emploi cette structure pour définir la particule marquant l'irréalisation d'un fait.

${ }^{4}$ Badawi, El-Sayyid, Carter, Michael et Gully, Adrian, Modern Written Arabic : A comprehensive Grammar, Londres / New York, Routledge, 2004, p. 645.

${ }^{5}$ Dans les exemples repris des grammaires, nous faisons précéder la traduction des auteurs puis traduisons en français leur traduction.
} 
C'est cette même et unique interprétation contrefactuelle que Buckley ${ }^{6}$ illustre par trois exemples.

(2) Allāhu waḥdahu ya lamu kam kuntu sa-uqāsī law kuntu fī baladihi wa-lī țiflun minhu.

"God alone knows how much I would have suffered had I been in his country and had a child by him. »

«Dieu seul sait combien j'aurais souffert si j'avais été dans son pays et j'avais eu un enfant de lui. »

(3) Lam yakun bi-wus 'ī an ahssara l-ṣafqata wa-kuntu sa-ahssaruhā idāa taḩallaftu. « I could not lose the deal, and I would have lost it if I had not shown up. » «Je ne pouvais pas perdre la transaction, et je l'aurai perdue si je m'étais absenté. »

(4) Wa-lākinna ḥālì ka-šabaḥin afḍalu mimmā kuntu sa-akūnu 'alayhi law 'araftu hayayan mā hum 'alayhi min kadibin

« But my state as a ghost is better than it would have been if I had known while I was alive what liars they are. »

« Mais mon état de fantôme était préférable à ce que j'aurais été si j'avais su, quand j'étais vivant, combien ils lui avaient menti.»

Tout comme dans (1), les occurrences de ces trois exemples sont liées au système hypothétique. La structure apparaît ici dans l'apodose antéposée de phrases conditionnelles marquant l'irréel du passé, cas sur lequel nous reviendrons plus bas.

En 2005, on retrouve cet emploi dans une autre grammaire d'arabe moderne, celle de Ryding ${ }^{7}$, où figurent trois nouvelles attestations, toujours contrefactuelles.

\footnotetext{
${ }^{6}$ Buckley, Ronald, Modern Literary Arabic : A Reference Grammar, Beyrouth, Librairie du Liban, 2004, nouvelle édition 2007, p. 562. Il n'explique pas la structure en elle-même, mais décrit en termes traduits : "In hypothetical sentences, kāna + sa- + imperfect indicative gives the meaning "would have" " (dans des propositions conditionnelles, kāna + sa- + l'inaccompli indicatif donne le sens de "aurait" "). Les trois exemples sont du même auteur, Gāala l-Sammān (al-Qamar al-murabba ${ }^{\prime}$ ).

${ }^{7}$ Ryding, Karin, A Reference Grammar of Modern Standard Arabic, Cambridge, Presses Universitaires de Cambridge, $2005,3^{\text {ème }}$ édition, 2007, p. 449 trouve une formulation qui nous semble assez juste : « to describe an action that would or could have taken place, but actually did not, the past tense of kān-a is used with the future tense of
} 
Dans le premier exemple, le contexte fait défaut pour décrire convenablement l'occurrence, car on ne sait pas s'il s'agit d'une conditionnelle tronquée ou d'un emploi indépendant:

(5) Mā kānat sa-ta 'rifu l-qirā'a wa-l-kitāba.

«She would not have known [how] to read and write. » « Elle n'aurait pas su lire et écrire.»

Dans un autre exemple, l'occurrence apparaît là encore dans l'apodose antéposée d'une conditionnelle de type irréel du passé ${ }^{8}$ :

(6) Kāna l-farīq sa-yafūz bi-l-musābaqāt idā kānat al-lağna qad samahat lahum bi-t-tasābuq.

«The team would have won in the competitions if the committee had permitted them to participate. »

" L'équipe aurait gagné les concours si le comité l'avait autorisé à participer. »

La nouveauté réside dans le dernier exemple ci-dessous, davantage un futur proche entrant dans le cadre d'un passé qu'un simple contrefactuel, d'où la traduction en «was going to»(«il allait utiliser»), mais une fois encore, l'exemple est reproduit dans un contexte trop restreint pour permettre un développement ${ }^{9}$ :

(7) Kuntu sa-astahddim šay'an āḩar.

« I was going to use something else. »

«J'allais utiliser autre chose. »

Si l'on s'en tient aux seules attestations des grammaires, on est donc amené à penser que la structure kāna sa-yaf'alu (1) n'apparaît que dans des contextes liés, intégrant un

the main verb» (« pour décrire une action qui aurait ou pourrait avoir eu lieu, mais qui, de fait, n'est pas, on emploie le passé de kān-a avec le futur du verbe principal »).

${ }^{8}$ Il est d'ailleurs intéressant de noter que la particule employée ici est id attendre dans le cas d'un irréel du passé. Cet emploi ne semble pas exceptionnel en arabe contemporain.

${ }^{9}$ Il pourrait tout à fait s'agir d'une apodose dont la protase n'aurait pas été mentionnée, auquel cas l'on pourrait comprendre cette phrase comme «j'aurais utilisé autre chose, si... ». 
système hypothétique et (2) n'accepte qu'une interprétation de type contrefactuel, comme irréel du passé ${ }^{10}$, à l'exception d'une mention d'un emploi de futur proche dans le passé (toutes réserves faites sur cette attestation où l'absence de contexte ne permet pas de garantir cette interprétation). Or, l'observation de notre corpus révèle que de nombreux emplois figurent en dehors d'une proposition conditionnelle. Aussi, un usager averti des grammaires d'arabe moderne est en droit de se demander si ces exemples sont représentatifs de la réalité d'emploi de cette structure en arabe moderne et si elles recensent toutes ses valeurs. La structure kāna sa-yaf alu ne peut-elle pas, comme le français «il allait faire » et l'anglais « he was going to do », supporter une interprétation factuelle de type « futur historique » ou encore d'autres valeurs ${ }^{11}$ ?

\section{Le corpus : présentation des occurrences}

Pour faire le point sur l'utilisation de cette structure en arabe moderne, nous décidons de l'observer systématiquement dans un corpus d'arabe contemporain afin d'en fournir une description et d'en classer les différents emplois ${ }^{12}$. Le corpus en question contient 94 occurrences de la structure kāna sa-yafalu ${ }^{13}$ :

\section{- 12 emplois sont dialectaux :}

- 7 proviennent du syrien (6 kān 'am yaf'al / 1 rāḥ yaf'al) $)^{14}$

- 5 sont en égyptien (kān ḥa-/ha-yaf'al)

Ces emplois dialectaux ne seront pas traités dans cet article consacré à l'arabe standard contemporain.

\footnotetext{
${ }^{10} \mathrm{Cf}$. à ce sujet Sartori, Manuel, " Pour une approche relationnelle de la conditionnelle en arabe littéraire moderne ", Arabica LVII, 2010, p. 79.

${ }^{11} \mathrm{Par}$ «futur historique», nous voulons désigner une forme verbale future employée pour parler d'un événement postérieur à un autre événement passé dont on parle au passé («La Première Guerre mondiale éclata en 1914. Elle allait durer / durerait 4 ans »), c'est-à-dire un futur dans le passé avec une interprétation factuelle.

${ }^{12}$ Corpus original d'1,5 million de mots, regroupant des textes produits entre 2002 et 2011, provenant de 7 pays arabes (Maroc, Tunisie, Égypte, Syrie, Liban, Arabie Saoudite, Yémen) et ressortissant à 3 genres (littérature, presse, blogs).

${ }^{13}$ Dans Pinon, Catherine, La nébuleuse de kān: classification des différents emplois de kāna / yakūnu à partir d’un corpus d'arabe contemporain, dir. Pierre Larcher, Aix-en-Provence, thèse de doctorat inédite, 2012 (désormais Pinon, Nébuleuse) et Larcher, Occurrence, il est fait mention de 99 occurrences, mais des doublons ont été retirés.

${ }^{14}$ Formellement, il s'agit dans les deux cas d'un inaccompli précédé d'une particule entrant dans le champ de kāna. Sémantiquement, il existe une différence entre les deux structures, kān 'am yaf'al traduisant plutôt « il était en train de » et kān rāh yaf'al « il était sur le point de / a failli ».
} 


\section{- 36 emplois sont syntaxiquement libres ${ }^{15}$}

- 46 correspondent à des emplois liés ${ }^{16}$, où sa-yafalu est dans le champ de kāna, lui-même lié à une particule qui le détermine. Ces particules sont:

- id $\underline{d} \bar{a}: 6$ (protase d'un système hypothétique), 1 (interrogative indirecte)

- id $\underline{d} \bar{a} m \bar{a}: 2$ (interrogation indirecte)

- in : 7 (protase d'un système hypothétique), 6 (interrogation indirecte), 4 (concession)

- law : 14 (apodose d'un système hypothétique), 2 (concession), 1 (protase d'un système hypothétique)

- wa-illā fa- : 2 emplois

- ammā : 1 emploi

\section{Analyse et résultats}

4. 1. Kāna sa-yaf'alu réanalysable comme futur dans le champ d'une particule composée avec kāna [(particule-kāna) sa-yaf'alu]

Dans ce cas, kāna est dépourvu d'incidence sémantique car son apparition est conditionnée par le contexte précédent. Il joue un rôle purement syntaxique et, de fait, sa rencontre avec sa-yaf'alu est pour ainsi dire fortuite. Il s'agit alors toujours d'une occurrence en contexte lié, c'est-à-dire induite par une particule précédente.

\section{1. 1. Dans une interrogation indirecte}

Dans le cadre d'une interrogation indirecte, kāna sa-yaf'alu ne doit pas être analysé comme une structure, mais plutôt décomposé comme un futur (sa-yaf'alu) entrant dans le champ d'une particule d'interrogation indirecte (id

\footnotetext{
${ }^{15}$ Pour une définition des emplois syntaxiquement libres, cf. infra 4.2.

${ }^{16}$ Pour une définition des emplois syntaxiquement liés, cf. infra 4.1.
} 
une valeur purement syntaxique ${ }^{17}$. Nous avons 9 emplois de ce type ( 1 avec 'ammā id $\bar{a}, 2$ avec id $\underline{d} \bar{a} \bar{a}$ et 6 avec in $)^{18}$. Dans ce cas, si l'emploi de kāna est syntaxique, il semble avoir deux origines distinctes. En effet, dans les deux exemples avec id deux de blogs, l'un marocain, l'autre saoudien, le recours à kāna permet d'enchâsser une phrase nominale, autrement dit de mettre l'accent sur le thème de l'interrogation. Kāna permet de conserver la structure thématique de la proposition enchâssée ${ }^{19}$ :

(8) Blog Marrokia (Maroc) ${ }^{20}$

Āh min al-rusūm al-mutaḥarrika. Aštāqu li-ayyām al-ṭufūla ḥaqqan, ahinnu li-kurat al-kabtin māğid al-latī taẓall fī al-hawā' yawman kāmilan, fa-naẓallu ma a-hā naḥnu fī intizāâr ma rifat id̄ā mākāna [sic] Ra'd al-mutamarrid sawfa yasmahuu bi-duhūlihā li-marmāh aw lā.

«Ah, les dessins animés... Je regrette vraiment l'époque de l'enfance, je suis nostalgique [quand je pense] au ballon du Capitaine Māğid qui pouvait rester suspendu dans les airs une journée entière alors que nous, nous étions avec lui dans l'attente de savoir si Tonnerre-le-rebelle allait le laisser entrer dans ses cages ou pas. »

On serait donc tenté de dire ici que kāna remplit l'une de ses fonctions classiques. Il est tout aussi intéressant de remarquer que la particule $m \bar{a}$ et le verbe kāna sont graphiquement associés en un seul mot (idā mākāna).

En revanche, dans les autres interrogatives indirectes, le verbe au futur apparaît directement après la particule interrogative composée in kāna, comme :

\footnotetext{
${ }^{17}$ Pour Badawi, Carter et Gully, Modern, p. 714, ida s'emploie davantage que in après le verbe sa'ala (demander) ou un verbe équivalent pour introduire une interrogative indirecte. Dans notre corpus, c'est l'inverse qui apparait à proportion de deux tiers contre un tiers.

${ }_{18} 5$ proviennent de blogs, 3 de littérature et 1 de la presse ; 3 sont yéménites, 2 saoudiens, 1 syrien (presse), 1 égyptien (blog), 1 libanais et 1 marocain.

${ }^{19}$ Ce rôle de focalisation a été décrit et documenté, notamment dans Larcher, Pierre, "Y-a-t-il un auxiliaire verbal en arabe ?», dans Jean-Louis Duchet, L'Auxiliaire en question, Travaux linguistiques du CERLICO, 2, Rennes, Presses Universitaires de Rennes, 1990 et Pinon, Catherine, «Les valeurs de kāna en arabe contemporain», Romano-Arabica XIII, Arab Linguistic, Literary and Cultural Studies, Bucarest, Presses de l'Université de Bucarest, 2013, p. 317-318, à la suite de Pinon, Nébuleuse, p. 107-109.

${ }^{20}$ Posté le 09/12/2010 ; en ligne [http://www.marrokia.com/2010/12/blog-post_09.html]. Le passage fait allusion à l'adaptation arabe du dessin animé Olive et Tom, Tonnerre le rebelle étant le gardien de but de l'équipe adverse de celle du Capitaine Māğid.
} 
(9)

Blog Swalfy (Arabie Saoudite) $)^{21}$

Sa-urāsilu-hum ba'da qalīl wa-arā in kānū sa-yaruddūna 'alayya wa-kayfa sayakūnu raddu-hum.

«Je leur écrirai sous peu pour voir s’ils me répondront et quelle sera leur réponse. »

En arabe, in doit être suivi d'un verbe ${ }^{22}$. Or, sa-yaf'alu en est un. On peut alors s'interroger sur l'insertion de kāna entre in et sa-yaruddūna dans (9). Pourrait-on, dans ce cas, s'en passer?

(9') ? Sa-urāsilu-hum ba 'da qalīl wa-arā in sa-yaruddūna 'alayya...

Il est en fait tout à fait inhabituel de voir un verbe précédé de sa-entrer dans le champ de in. Ici, il semble que kāna soit utilisé pour faire tampon entre la particule in généralement suivie d'un accompli ou d'un apocopé, et le verbe en sa-yaf'alu. Son emploi est donc toujours syntaxique, mais pas pour les mêmes raisons que celles qui avaient expliqué sa présence après id $\underline{a} \bar{a} \bar{a}$. Ainsi, si les emplois de la particule in sont amenés à se diversifier, comme ici en acceptant dans son champ un futur formel, les contraintes séculaires quant à la forme de ce que cette particule peut intégrer dans son champ s'appliquent encore aux réalisations actuelles.

Si l'on est tenté de faire l'hypothèse que ces constructions d'interrogatives indirectes sont un calque de langues européennes, force est de constater que les deux raisons d'emploi de kāna dans ces structures sont bien propres à la langue arabe. D'autre part, que sur si peu d'attestations les 3 genres soient représentés ainsi que 6 pays sur les 7 que compte notre corpus, suggère que ce phénomène est bien intégré au domaine arabe.

\footnotetext{
${ }^{21}$ Posté le 11/10/2006; en ligne [http://swalfy.com/date/2006/10]

${ }^{22}$ Après in, on trouve toujours un verbe, qu'il soit à l'apocopé, à l'accompli ou qu'il s'agisse de kāna employé pour des raisons syntaxiques. Les cas où in n'est pas directement suivi d'un verbe résultent d'une ellipse. En effet, l'ellipse de kāna, avec son ism, est possible après les particules hypothétiques in et law, pour alléger la formulation. Les deux exemples les plus cités par les grammairiens arabes sont souvent repris par les orientalistes dans leurs grammaires : "An-nāsu mağziyyūna bi-a māli-him in hayran fa-ḩayrun wa-in šarran fašarrun... » («Les gens sont récompensés pour leurs actions : si elles sont bonnes, [ils le sont en] bien, et si elles

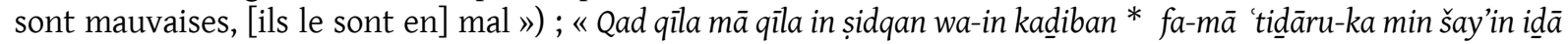
qïlā ?» («Ce qui a été dit a été dit, que ce soit vrai ou faux * que [vaut] votre excuse face à une chose quand elle a été dite?»).
} 
Pour Badawi, Carter et Gully, les deux types d'interrogations indirectes sont des calques de langues européennes. Pour la particule in, ils fournissent des exemples soit de phrase nominale enchâssée, soit de phrase verbale où le verbe enchâssé est un inaccompli. Avec i $\underline{i} \bar{a}$ en revanche, nous trouvons des attestations formellement semblables aux nôtres. L'une d'entre elles a particulièrement attiré notre attention; elle apparaît dans un paragraphe intitulé «conditionals in reported speech» («conditionnelles dans le discours rapporté »), où les auteurs notent que " use of kāna "be" with an independent imperfect verb in the protasis to denote the past ${ }^{23}$ :

(10) Lā a rifu iḍā kāna hād̄ā țabī'a fī-hā am li-annahā lā tunğibu

«I do not know whether this was a peculiarity in her or [whether it was] because she is not producing children ".

"Je ne sais pas si c'était une nature chez elle ou si c'était parce qu'elle n'avait pas eu d'enfants. "

Deux remarques s'imposent : tout d'abord, les auteurs interprètent sémantiquement kāna comme un passé, là où nous pensons qu'il s'agit d'un emploi syntaxique lié à id̄ā. En effet, il est tout à fait possible de comprendre cette phrase comme une généralité :

(10') « Je ne sais pas si c'est une nature chez elle ou si c'est parce qu'elle n'a pas d'enfants ».

Si le locuteur avait vraiment voulu mettre son interrogation au passé, n'aurait-il pas accordé kāna avec tabī' $a$, d'une part, et n'aurait-on pas eu plutôt lam tunğib d'autre part ? ${ }^{24}$ Peut-être que, partant du principe que cette structure est forcément un calque des langues européennes, les auteurs l'interprètent comme imitant, en arabe, la syntaxe de l'anglais. Si leur interprétation était correcte, il faudrait alors relever une évolution: l'emploi de l'accompli dans le champ de id $\underline{\text { a }}$ pour dénoter un passé et non plus comme jouant un rôle formel et exprimant le présent.

Ensuite, nous sommes perplexe devant l'emploi du terme « conditionnelle » pour ce type de

\footnotetext{
${ }^{23}$ Badawi, Carter et Gully, Modern, p. 670 : «kāna est employé avec un inaccompli dans la protase pour dénoter le passé »

${ }^{24}$ L'absence d'accord est en effet un argument fort en faveur du développement de id̄ākān, ici écrit en un seul mot, comme étant une particule du conditionnel.
} 
phrase. Pour nous, la particule a clairement évolué dans le sens d'un simple marqueur d'interrogation indirecte. S'agirait-il d'une nouvelle valeur qui tarderait à intégrer les grammaires ou doit-on simplement voir dans cette appellation une certaine emprise des grammaires classiques sur les linguistes ? Pour juger cette valeur nouvelle, il faudrait étudier des textes classiques pour voir si elle en est bien absente.

Chez Buckley (Modern, p. 687), bien que les exemples qui nous intéressent se rattachent au chapitre sur les interrogatives, les particules idā et in sont aussi nommées « conditional particles» («particules conditionnelles»). Celui-ci note que « one of the most common uses of in in modern Arabic is to introduce an indirect question ("whether", “if"). $»^{25}$ Les huit exemples qu'il donne commencent par 'in kān. Pourquoi dans ce cas faudrait-il continuer à nommer in « particule conditionnelle»?

\section{1. 2. Dans une concession}

Nous avons dans notre corpus 6 propositions concessives ${ }^{26} .3$ sont de la forme wa-in kāna, 1 hattāa wa-in kāna, 1 wa-law kāna, 1 hattā law kāna. Ces différentes manières d'introduire une concessive sont toutes mentionnées par Badawi, Carter et Gully, Modern, p. 650-651, 669.

(11) Blog Harkasha (Syrie) ${ }^{27}$

Hunāka marḥala intahat wa-uḩrā tabda' al-āna fī kull makān min 'ālam al-'arab, wain kāna taḥqīquhā sa-yatimm bi-sur'āt muhtalifa wa-aškāl mutabāyina, hunā aw hunāk.

«Une étape a pris fin, une autre commence maintenant dans tout le monde arabe, même si le passage d'une étape à une autre se fera à des vitesses et sous des formes différentes selon l'endroit. »

Dans le cas des concessives, il est préférable d'analyser ces occurrences comme étant l'ajout d'un kāna à la particule concessive comprenant dans son champ un futur en sa-

\footnotetext{
${ }^{25}$ Buckley, Modern, p. 734. "L'un des usages les plus courants de 'in en arabe moderne est d'introduire une question indirecte ("si"). » "Si" traduit les deux termes anglais cités « whether », « if ».

${ }^{26} 5$ de ces occurrences proviennent de blogs, une seule de la presse. Deux occurrences sont égyptiennes, deux autres syriennes, 1 yéménite et 1 saoudienne.

${ }^{27}$ Récupéré à l'adresse [http://karkasha.com/] mais ce blog n'existe plus en 2014, probablement pour des raisons politiques.
} 
yaf'alu. Nous observons les mêmes phénomènes que pour les interrogatives indirectes, à savoir le recours à kāna après la particule soit pour permettre la focalisation sur le thème, soit pour introduire un futur dans le champ de particules qui n'en acceptent généralement pas. Il s'agit, là aussi, d'un phénomène plus général, devenant peut-être un « réflexe » chez certains locuteurs arabes, où l'on ajoute systématiquement kāna après une particule.

\section{1.3. Dans la protase d'un système conditionnel}

Nous avons 15 occurrences de kāna sa-yaf'alu en protase d'un système hypothétique : 7 de la forme in kāna sa-yaf'alu, 6 de la forme id yaf'alu et ammā kāna sa-yaf'alu. Ici encore, il convient de réanalyser l'amalgame particulekāna sa-yaf'alu comme un futur formel sa-yaf'alu entrant dans le champ d'une particule conditionnelle à laquelle le verbe kāna est adjoint pour des raisons syntaxiques. Ce phénomène, déjà mis en avant ici, est décrit dans différents ouvrages ou publications ${ }^{28}$. Il en va de même pour l'occurrence de ammā kāna sa-yaf'alư ${ }^{29}$.

Nous avons relevé dans notre corpus au moins une attestation de la structure kāna lan yaf'al, argument fort en faveur de l'analyse syntaxique que nous proposons, à savoir le rattachement de kāna à la particule idāa, comprenant dans son champ cette fois-ci la négation formelle d'un futur.

Presse : An-Nahār (Liban) ${ }^{30}$

Fa-ịā kānat al-nahḍa bi-maḍụūni-hā al-tanmawī lan tuhaqqiqa-hā baladiyyāt altawāfuq bayna quṭbay al-sāḥa al-šīi ìyya fa-man yuhaqqiqu-hā ?

«Si le renouveau, dans sa dimension de développement, n'est pas réalisé par les municipalités de coalition des deux pôles du domaine chiite, alors qui le réalisera?»

4. 2. Kāna sa-yaf'alu analysable comme une même structure [kāna sa-yaf'alu]

\footnotetext{
${ }^{28}$ Notamment Pinon, Valeurs. Pour d'autres références, $c f$. Pinon, Nébuleuse, p. 122 et suiv.

${ }^{29}$ Sur ammā et immā,$c f$. Pinon, Nébuleuse, p. 39.

${ }^{30}$ Article désormais accessible dans les archives payantes du journal [www.annahar.com], en date du 12 avril 2011.
} 
Dans ce cas, kāna sa-yaf'alu est une construction spécifique portant une valeur contrefactuelle. Cette structure peut apparaitre dans des contextes syntaxiques divers : soit dans un contexte syntaxiquement non contraint (que nous avons appelé «syntaxiquement libre »), soit en contexte lié, kāna sa-yaf'alu apparaissant dans ce cas uniquement dans l'apodose des systèmes hypothétiques. Nous renvoyons aux exemples analysés par Larcher dans Occurrence et en proposons ici de nouveaux.

\section{2. 1. Dans l'apodose d'une conditionnelle}

Nous avons 16 occurrences de kāna sa-yaf'alu dans l'apodose d'une conditionnelle : 2 fois après wa-illa $f a-$ et 14 fois dans l'apodose d'une conditionnelle en law. Dans le cas de ces dernières, 4 propositions présentent l'ordre law $p, q$ ( $p$ symbolisant la protase et $q$ l'apodose) alors que 10 autres ont préféré l'antéposition de l'apodose, soit l'ordre $q$, law $p$.

L'étude des occurrences du type law $p, q$ s'avère intéressante, car on se demande pourquoi le locuteur recourt à la forme kāna sa-yaf alu pour marquer un fait irréalisé alors que l'emploi de la particule law apporte déjà cette valeur.

(13) Blog Insana (Égypte) ${ }^{31}$

Wa-ḩtartu l-ḥall al-țānī li-annī law kuntu -nsahabtu kuntu sa-ạ̣zanu katīiran.

«J'ai choisi la seconde solution, parce que si j'avais abandonné, j'aurais été très triste. »

Pour nous, cette phrase est sémantiquement équivalente de celle-ci :

(13’) Wa-ḥtartu l-ḥall al-țānī li-annī law kuntu -nsahabtu la-ḥazintu katīiran.

Il est donc légitime de s'interroger sur le processus qui amène kāna sa-yaf'alu dans l'apodose du système en law. Nous pouvons supposer que la structure kāna sa-yaf'al qui a en contexte non lié valeur d'irréalisé, intègre les apodoses des systèmes en law exprimant eux aussi

\footnotetext{
${ }^{31}$ Publié le 31/05/2010 à l'adresse [http://2insana.blogspot.fr/2010/05/blog-post_31.html].
} 
l'irréalisé. La redondance sémantique ne se justifierait que par le désir d'employer une forme qui, aux yeux du locuteur, exprime par excellence l'irréalisé : kāna sa-yaf'al. D’autre part, on remarque dans ces quatre conditionnelles en law que le lām d'apodose n'apparaît jamais. Ainsi, le rôle de kāna serait aussi celui de segmentateur marqueur d'apodose. En effet, dans notre corpus, les seules attestations d'un système hypothétique en law où le lām de l'apodose fait défaut présentent une apodose en kāna sa-yaf'alu. Dans ce cas, aucun autre segmentateur comme fa-(inna) par exemple n'apparaît non plus, ce qui est un argument fort pour considérer kāna dans ce cas comme un segmentateur ${ }^{32}$. Cependant, les occurrences sont trop peu nombreuses pour permettre d'affirmer cela avec certitude.

Dans le cas où l'apodose est antéposée à la protase, nous remarquons que kāna sayaf'alu peut supporter différents degrés dans l'échelle du contrefactuel. Dans ce premier exemple, nous sommes dans le contrefactuel le plus total :

Presse : Al-Tağdīd (Maroc) ${ }^{33}$

[...] akkadū [...] bi-anna kārița ḥaqīqiyya kānat sa-taḥullu bi-ḥayyi al-Amal law tazāmana ḥādit al-inhiyār ma 'a awqāt al-ṣalāt.

«[...] ils ont insisté [...] sur le fait qu'une véritable catastrophe serait survenue dans le quartier al-Amal, si l'effondrement avait coïncidé avec les heures de prière. »

Law exprime une hypothèse irréalisable et irréalisée: l'effondrement ayant eu lieu en dehors des heures de prière, la catastrophe a été évitée. L'apodose exprime donc un fait qui ne s'est pas produit et ne pourra pas se produire.

Parfois, si l'apodose entre logiquement dans l'irréalisable, le degré de contrefactualité de l'apodose n'est pas le même, comme dans l'exemple suivant:

Littérature : Naji Ṭāhir (Liban) ${ }^{34}$

\footnotetext{
${ }^{32}$ Dans Larcher (à paraître), l'exemple (2) exhibe un kāna sa-yaf'al dans le champ de fa-inna-hā. De fait, kāna est la forme requise par le système hypothétique en law et dans notre exemple (13), l'interprétation en tant qu'irréel du passé est belle est bien assurée par la protase de la forme law kāna fa 'ala: «law kuntu-nsahabtu ». Une apodose en kāna sa-yaf'al apparaitt donc comme un trait post-classique, sémantiquement redondant.

${ }^{33}$ Publié le 01/03/2010 à l'adresse [http://www.attajdid.info/] ; désormais accessible à l'adresse [http://www.maghress.com/hibapress/805].

${ }^{34}$ Nouvelle écrite en 2006, intitulée Mală'ikat al-adrāğ, mise en ligne le 30 juin 2007 à l'adresse [http://www.arabicstory.net/?p=text\&tid=10413].
} 


\section{Mādā kunta sa-taf'al ! Law rağa'a l-zamānu sā'a !}

«Que ferais-tu, si tu revenais une heure en arrière?»

Dans cet exemple, comme dans les conditionnelles en law de manière générale, le fait exprimé dans la protase est irréalisé. Mais dans l'apodose, même si le fait est logiquement irréalisable, l'interlocuteur peut tout à fait exprimer son opinion et dire ce qu'il ferait. On est donc dans du contrefactuel du fait que la condition exprimée dans la protase soit impossible à réaliser, mais sur le plan de l'imaginaire, de l'expression de l'opinion, cet acte irréalisable peut être exprimé. D'ailleurs, ce qui confère à l'apodose une valeur potentielle, c'est que l'antéposition qui amène à comprendre d'abord kāna sa-yaf'al comme indépendant est renforcée par la séparation matérielle des deux composantes de la conditionnelle opérée par le point d'exclamation. C'est pourquoi nous avons traduit par du conditionnel présent et de l'imparfait, et non pas par du conditionnel passé et du plus-que-parfait (« qu'aurais-tu fait, si tu étais revenu une heure en arrière? »). On pourrait pousser, et le contexte le permettrait dans ce cas, jusqu'à comprendre cet exemple comme deux propositions syntaxiquement indépendantes, la première étant une interrogative, la seconde une optative :

(15') «Qu'allais-tu faire ? Si seulement on pouvait revenir une heure en arrière... »

La ponctuation en arabe semble d'ailleurs montrer qu'il s'agit de la bonne interprétation.

Un autre exemple nous amène à réfléchir au lien qui pourrait exister entre l'emploi de kāna sa-yaf'alu et l'antéposition de l'apodose. Prenons un exemple : dans un blog saoudien, l'auteur donne son avis sur une affiche publicitaire pour une entreprise spécialisée dans la création de sites web. Il dit:

(16) Blog Jihād (Arabie Saoudite) $)^{35}$

Kalimatay [sic.] "Fancy” wa "Web Sites” bārizatayni [sic.] wa-kānatā sa-yu abbirāni 'an našāṭ al-maktab bi-fā' iliyya law -iqtașarat al-lawḥa 'alayhimā.

"Les mots "Fancy" et "Web sites" ressortent [mais] auraient exprimé efficacement le dynamisme du bureau si l'affiche s'était réduite à ces deux mots. »

\footnotetext{
${ }^{35}$ Posté le 29/12/2006 à l'adresse [http://www.blogjihad.com/?m=200612]. Mêmes références pour (16).
} 
Au début, on serait tenté d'analyser wa-kānatā sa-yu 'abbirāni comme un potentiel : "elles exprimeraient mieux », lecture sur laquelle on revient quand on arrive à la protase qui nous oblige à re-analyser comme un irréel, étant donné que cette protase présuppose que l'affiche ne se réduit pas à l'expression de ces deux mots. L'auteur argumente pour convaincre le lecteur que l'affiche serait mieux comme il l'imagine lui, et l'antéposition de la protase s'avère pragmatiquement efficace, car son argument est plus persuasif puisqu'il laisse à penser au lecteur que la modification est possible et améliorerait l'affiche. Un peu plus loin, on peut percevoir le même phénomène (notons au passage que le verbe entrant dans le champ de kāna est kāna lui-même), où l'argumentation se trouve renforcée par le verbe introducteur ya 'taqid:

(17) Blog Jihād (Arabie Saoudite)

A taqid annahu kāna sa-yakūnu afḍal bi-katīi law lam yakun fī l-lawḥa illā lkalimatayni l-bārizatayni [sic.], wa-'unwān al-mawqi '[...]

«Je crois que ce serait [> ça aurait été] beaucoup mieux si n'avaient figuré dans l'affiche que les deux mots et l'adresse du site [...]»

Il arrive aussi, dans un exemple au moins, que law n'introduise plus une condition irréalisable et irréalisée, mais qu'elle introduise une condition plus ou moins probable, comme dans l'exemple suivant, dont l'interprétation peut être double :

(18) Presse : Al-Wațan (Syrie) ${ }^{36}$

[...] wa-ašāra ilā anna l-sā’iqīna kānū sa-yaltazimūna bi-l-'addādāt law tamma taḩfị̣ si r al-banzīn aw l-ḍarā’ib lākin wa-fī zill g̉iyāb dālika fa-innā min haqquinā ziyāda dahlinā aswatan bi-ġayrinā.

«[...] il a indiqué que les chauffeurs [de taxi] auraient utilisé les compteurs si le prix du carburant ou des taxes avait diminué, mais qu'en l'absence [d'une telle baisse], nous avons le droit d'augmenter notre revenu comme les autres. »

Il s'agit d'un article qui s'interroge sur les raisons qui poussent les chauffeurs de taxi qui utilisent les compteurs à réclamer systématiquement au moins 10 Livres de plus que le prix

${ }^{36}$ Publié le 13/04/2011 à l'adresse [http://www.alwatan.sy]. Désormais en ligne à cette adresse [http://www.dp-news.com/pages/detail.aspx?articleid=80641]. 
indiqué. Le chauffeur de taxi interrogé répond que c'est pour eux le seul moyen d'augmenter leurs revenus, comme arrivent à le faire les citoyens d'autres corps de métiers. Dans cette phrase, vu que les baisses de prix ou de taxes n'ont pas eu lieu, c'est l'irréel qui se trouve clairement exprimé. Mais on peut aussi comprendre cette phrase autrement:

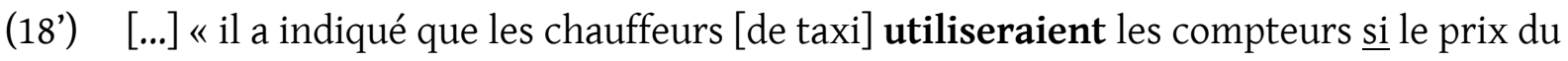
carburant ou des taxes diminuait, mais qu'en l'absence [d'une telle baisse] [...] »

Sémantiquement, par rapport au contexte, cette interprétation est possible. Une baisse du prix du carburant ou des taxes relevant d'un choix politique, on ne peut pas l'exclure totalement. Dans ce cas, il faudrait accepter que law introduise une conditionnelle potentielle, réalisable. La particule «empièterait» alors sur les valeurs habituellement dévolues aux particules in et $i \underline{d} \bar{a}$ et sortirait du champ de l'irréel pour englober celui du potentiel $^{37}$. Est-ce là un trait d'évolution de l'arabe contemporain ? Ainsi, l'emploi de kāna sa-yaf'alu, ayant dans les contextes non liés une valeur de conditionnel potentiel, attirerait law dans le champ du potentiel. Autrement dit, la valeur de la structure kāna sa-yaf'alu syntaxiquement libre finit-elle par recouvrir les autres valeurs qu'elle devait posséder dans les contextes liés? Voilà encore une hypothèse qui mériterait d'être soumise à une étude de large envergure.

\section{2. 2. Occurrences syntaxiquement libres}

Nous avons relevé 36 occurrences d'un emploi de kāna sa-yaf'alu syntaxiquement libre, mais faisant sémantiquement toujours partie du champ de l'hypothétique. En effet, même lorsqu'il s'agit de futur (proche) dans le passé, kāna sa-yaf'alu sert à exprimer des actions qui ne se sont pas réalisées, quelles qu'en soient les raisons (qu'il s'agisse d'un prérequis non réalisé, d'un phénomène impossible ou encore d'un propos imaginaire). Nous présentons d'abord le principal emploi où, dans un schéma logique récurrent, le recours à kāna sa-yaf'al permet de croiser deux relations logiques. Dans l'exemple suivant, le contexte est élargi pour permettre une compréhension globale du processus :

\footnotetext{
${ }^{37}$ C'est le cas dans de nombreux dialectes. Pour l'égyptien, cf. Sartori, Manuel, «L'évolution des conditionnelles en arabe égyptien contemporain », BEO 58, 2009, p. 233-257.
} 
(19) Blog Shabayek (Égypte) $)^{38}$

'indamā qarrarat Lūrā al-taḍhiya bi-mașrūfihā l-šahrī fī 'ǐğār šāḥina tatawallā naql al-muhallafāt ilā mașāni ' i ādat tadwīr al-waraq wa-l-aluminyūm wa-l-zuğāğ wa-lblāstīk wa-ġayrihi, wa-stamarra 1-ḥāl 'alā ḍālika li-muddat 'āmayni, ḥattā annahu fī aḥad [sic.] al-marrāt naqalat al-šāḥina mā waznuhu 17 țunn min al-qumāma wa-llatī kānat immā sa-tuḥraq aw tulqā fī bāṭin al-arḍ li-tahtaliṭ bi- anāṣirihā wa-tusabbib al-talawwut bi-šatā șuwarihi, lākinnahā bi-faḍl Lūrā u'īda -stiȟdāmuhā li-tudhal fī șan ‘ ašyā’ uhrā mufīda li-l-insān wa-l-bī’a.

«Quand Laura[-Beth Moore] décida de sacrifier son revenu mensuel pour louer un camion pour s'occuper du transport des déchets à des usines de recyclage de papier, d'aluminium, de verre, de plastique et autre, cela a duré deux ans, si bien que lors d'un des passages, le camion a transporté 17 tonnes de déchets qui auraient soit été brûlées, soit enterrées, tout type de déchet confondu, ce qui aurait causé une pollution sous toutes ses formes, mais grâce à Laura, elles ont été réutilisées pour fabriquer d'autres objets utiles à l'homme et bons pour l'environnement. »

Le schéma syntaxique est le suivant : kāna sa- + affirmation yaf'alu + lākin (mais) + proposition affirmative. Dans cette proposition particulièrement complexe, l'affirmation introduite par kāna sa-yaf'alu oppose la réalité à ce qui aurait pu se passer si le fait exprimé dans la proposition suivant lākin «mais » ne s'était pas réalisé (dans l'exemple 18, les déchets auraient été brûlés ou enfouis si Laura n'avait pas payé de camion pour les mener à l'usine de recyclage). Autrement dit, lākin rectifie l'affirmation yaf'alu : le locuteur, tout en posant que l'état de faits décrit par l'affirmation aurait pu avoir lieu, pose qu'il n'a pas eu lieu. Il laisse donc présupposer que le fait qui impliquait la réalisation de l'état de faits qui aurait pu survenir ne s'est pas réalisé et qu'un autre fait, impliquant la conclusion logique inverse de la réalisation précédente, s'est lui réalisé. Lākin oppose deux conclusions logiques, l'une qui ne s'est pas réalisée (mais qui aurait pu se réaliser) car son inverse logique s'est réalisée. Ces deux conclusions, exprimées dans la proposition, présupposent deux arguments dont nous comprenons que l'un ne s'est pas réalisé alors que l'autre oui. Nous pouvons appeler cet emploi un «contrefactuel logique ${ }^{39}$, car même si la condition de réalisation est implicite, cette condition n'étant pas réalisée, le fait exprimé par kāna sa-

\footnotetext{
${ }^{38}$ Posté le 08/03/2011 à l'adresse [http://www.shabayek.com/blog/2011/03/08/].

${ }^{39}$ Sur l'emploi de kāna comme commutateur factuel / contrefactuel, cf. Pinon, Valeurs, p. 311.
} 
yaf'alu n'est pas réalisé.

Si l'on schématise, les deux relations logiques d'implications sont présupposées dans l'énoncé : $p$ implique $q$ et non- $p$ implique non- $q$. Dans l'énoncé, non- $p$ et $q$ sont donnés : non- $p$ implique la non réalisation de q. C'est la structure kāna sa-yaf'alu qui produit le sens de non réalisation. Ainsi, dans notre exemple, $p$ (avant que Laura n'intervienne) implique $q$ (les ordures étaient jetées) et non- $p$ (Laura intervient) implique non-q (les ordures sont recyclées). Dans l'énoncé, nous avons non-p (Laura intervient) qui implique kāna sa-yaf'alu $q$ (non réalisation de « les ordures sont jetées », mais ça aurait pu), lākin (« mais ») non q (les ordures sont recyclées, conclusion logique de non-p posé plus haut).

Ce schéma logique est attesté plusieurs fois au sein des occurrences étudiées, mais il y a encore d'autres degrés dans la contrefactualité :

(20) Blog Swalfy (Arabie Saoudite) $)^{40}$

Al-mabnā l-ğadīd allad̄ī tamma taḥwīluhu li-l-țullāb al-mustaǧiddīn huwa mabnā buniya asāsan li-takūn li-kulliyyat al-ḥāsib al-ālī lā adrī kayfa sa-yastafīdūna al-țullāb al-mustaǧiddūn min ma āmil țullāb handasat al-ḥāsib al-ālī -llatī kāna sa-yastafīd minhā țullāb hādāa l-taḩașṣuṣ bi-šakl kabīr bi-mā taḥtawīhi min tağhīzāt wa-adawāt li-taḩașṣuṣihim wa-lā adrī ayḍan kayfa sa-yastafīd al-țullāb al-mustaǧiddūn min ma'amil al-barmağa -llatī kāna sa-yastafīdu minhā țullāb qism 'ulūm al-ḥāsib badalan min al-ma āmil al-ḥāliyya l-mutīira li-l-šafaqa.

«Le nouveau bâtiment qui vient d'être attribué aux étudiants de première année est un bâtiment qui a été construit, à la base, pour revenir à la faculté d'informatique. Je ne sais pas comment les étudiants de première année tireront profit des laboratoires des étudiants de génie informatique, qui auraient davantage profité aux étudiants de cette spécialité du fait de son équipement en appareils et instruments pour leur spécialité. Je ne sais non plus comment les étudiants de première année profiteront des laboratoires de programmation qui auraient profité aux étudiants des sections de sciences de l'informatique au lieu des laboratoires actuels qui font pitié. »

L'auteur déplore que les nouvelles salles d'informatique soient réservées aux étudiants

\footnotetext{
${ }^{40}$ Publié le 12/01/2009 à l'adresse [http://swalfy.com/category/ğāmi ‘iyyāt]. Le mot transcrit en italique figure en arabe dans l'adresse.
} 
débutants alors qu'il semble évident qu'elles seraient plus profitables aux étudiants dont la spécialité est l'informatique. Implicitement, l'auteur dit donc que si l'on avait utilisé ces salles pour les étudiants dont c'est la spécialité, ils en auraient davantage profité; explicitement, il affirme que l'on a donné ces salles aux débutants et que le profit attendu n’est donc pas concrétisé. Il utilise kāna sa-yaf'alu pour exprimer cette irréalisation.

Comme nous l'avons constaté, le degré de contrefactualité et de fictivité varie d'un exemple à l'autre. Une dernière valeur attribuée à la structure kāna sa-yaf'alu est celle de futur (proche) dans le passé.

(21) Presse : Al-Tawra (Yémen) ${ }^{41}$

Fa-innā 'anāṣir infișāliyya taḩrībiyya musallaḥa [...] hāğamat sayyārat mu'assasat 14 ūktūbar li-l-ṣihāāa wa-l-țibā'a wa-l-našr wa-l-i'tidā' 'alā sā'iqihā wa-nahb mumtalakātihi 1-šahsṣiyya wa-murāfiqīhi wa-muṣādara wa-iḩrāq nusah̆ al-ṣaḥifa 1yawmiyya -llatī kānat sa-tuwazza' 'alā 14 muḥāfaẓa [...].

«Des éléments séparatistes subversifs armés [...] ont assailli la voiture de la Fondation 14 Octobre pour la presse, l'édition et la diffusion en attaquant le conducteur et en pillant ses biens personnels ainsi que ceux de ses compagnons, confisquant puis brûlant les journaux quotidiens qui allaient être distribués dans 14 gouvernorats [...]. »

Dans cet exemple, kāna sa-yaf'alu exprime bien une action qui allait être réalisée quand, dans le passé, un élément a empêché cette réalisation. Si cette expression du futur dans le passé semble différer des autres emplois, elle n'en partage pas moins avec eux la même sphère logico-sémantique, celle de l'irréalisé.

En effet, ce qui fait le lien entre toutes nos occurrences, c'est que l'action introduite par kāna sa-yaf'alu n'est pas réalisée. Les causes de la contrefactualité sont diverses : soit la condition de réalisation est physiquement, devrait-on dire nécessairement, impossible (exemple 14), soit elle était potentiellement réalisable, mais ne s'est pas concrétisée dans les

${ }^{41}$ Publié le 08/03/2010 à l'adresse [www.althawranews.net]. Désormais en ligne à l'adresse [http://www.almotamar.net/news/print.php?id=78736]. 
faits $(13,15,16)$, soit elle est fictive (17), soit enfin elle a été empêchée par un événement survenu dans le passé (20). Logiquement, toutes ces propositions peuvent être réécrites comme des conditionnelles. Mais la condition n'est plus exprimée, elle est présupposée. Il serait peut-être plus exact de dire que le fait que cette condition ne soit pas réalisée est présupposé dans l'énoncé. C'est sur la non-réalisation du fait qui devait logiquement se réaliser si la condition l'était elle aussi, que celui qui utilise la structure kāna sa-yaf'alu porte l'attention du discours. Le fait de dire kāna sa-yaf'alu q signifie que $q$ ne s'est pas réalisé, et présuppose que la condition $p$ nécessaire à la réalisation de $q$ ne l'a pas été non plus. Il s'agit alors non pas d'exprimer ce qui pourrait se passer si une condition était remplie (le propre d'une conditionnelle), mais bien d'exprimer ce qui ne s'est pas produit.

Une telle analyse explique qu'aucune valeur factuelle n'apparaît dans notre corpus, à l'inverse du français « il allait faire » ou de l'anglais « he was going to do » qui peuvent exprimer ce que l'on a appelé le futur historique. En effet, une telle valeur sort totalement du cadre de l'irréalisé que déclinent nos exemples en autant de nuances contrefactuelles. Kāna sa-yaf'alu est, au regard de nos occurrences, logiquement et sémantiquement incompatible avec l'emploi de type «futur historique » du français ou de l'anglais. Mais il n'est pas impossible qu'une telle évolution se produise; elle serait alors certainement initiée par l'usage répété de calques de langues européennes. Dans l'état actuel des choses, il apparaît que kāna sa-yaf'alu est bel et bien une structure inhérente à l'arabe, quoique relativement rare : son existence en dialecte, la répartition diatopique de ses emplois tout comme le fait que des attestations très anciennes existent en sont autant d'arguments.

\section{2.3. Répartitions diatopique et par genres de la structure kāna sa-yaf' $a l u^{42}$}

La répartition par genres montre clairement que l'emploi de cette structure est plus rare dans la presse que dans la littérature et les blogs :

\begin{tabular}{|c|c|c|}
\hline Blogs & Littérature & Presse \\
\hline $\mathbf{4 1}$ & $\mathbf{3 2}$ & $\mathbf{9}$ \\
\hline
\end{tabular}

\footnotetext{
${ }^{42}$ Le total des occurrences s'élève à 82 occurrences car les 12 occurrences dialectales ne sont pas prises en compte dans ces tableaux.
} 
Tableau 1 : Occurrences des emplois de kāna sa-yaf'al par genre

La répartition diatopique montre un grand écart entre le Liban et la Tunisie, mais la structure est néanmoins représentée dans l'ensemble des pays figurant dans notre corpus et l'on peut estimer qu'elle est donc employée dans l'ensemble du domaine arabe :

\begin{tabular}{|c|c|c|c|c|c|c|}
\hline Liban & Yémen & Arabie S. & Syrie & Maroc & Égypte & Tunisie \\
\hline $\mathbf{1 7}$ & $\mathbf{1 5}$ & $\mathbf{1 4}$ & $\mathbf{1 2}$ & $\mathbf{1 1}$ & $\mathbf{8}$ & $\mathbf{5}$ \\
\hline
\end{tabular}

Tableau 2 : Occurrences des emplois de kāna sa-yaf'al par pays

Nous proposons de fournir le détail des occurrences de cette structure, par genre, pays et contexte syntaxique.

\begin{tabular}{|c|c|c|c|c|c|c|c|c|c|c|c|}
\hline \multirow{2}{*}{$\begin{array}{l}\text { Type } \\
\text { d'emploi } \\
\text { syntaxique }\end{array}$} & \multicolumn{3}{|c|}{ Genre } & \multicolumn{7}{|c|}{ Pays } & \multirow{2}{*}{$\begin{array}{l}\text { Total des } \\
\text { occurrences }\end{array}$} \\
\hline & Lit. & $\mathrm{Bl}$. & Pr. & Arabie & Égypte & Liban & Maroc & Syrie & Tunisie & Yémen & \\
\hline libre & 18 & 16 & 2 & 6 & 1 & 6 & 8 & 3 & 3 & 9 & 36 \\
\hline lié & 14 & 25 & 7 & 8 & 7 & 11 & 3 & 9 & 2 & 6 & 46 \\
\hline \multicolumn{12}{|l|}{ Dont: } \\
\hline $\begin{array}{l}\text { Interrogative } \\
\text { indirecte }\end{array}$ & 2 & 5 & 1 & 2 & 1 & 0 & 1 & 1 & 0 & 3 & 8 \\
\hline Concessive & 0 & 5 & 1 & 1 & 2 & 0 & 0 & 2 & 0 & 1 & 6 \\
\hline Protase & 5 & 9 & 3 & 1 & 2 & 7 & 1 & 3 & 1 & 2 & 17 \\
\hline Apodose & 7 & 6 & 2 & 4 & 2 & 4 & 1 & 3 & 1 & 0 & 15 \\
\hline
\end{tabular}

Tableau 3 : Occurrences de kāna sa-yaf'al par contexte syntaxique

\section{Conclusion}

L'étude de près d'une centaine d'occurrences de la structure kāna sa-yaf'alu en arabe contemporain a permis de dresser un portrait précis et complet des emplois actuels. Elle a montré :

1) que cette structure doit être comprise tantôt comme l'association du verbe kāna d'emploi 
syntaxique à une particule comprenant dans son champ un futur formel sa-yaf'alu, pour le cas des protases de conditionnelles, des interrogatives indirectes et des concessives, tantôt comme une structure indépendante, apparaissant alors en apodose de certains systèmes hypothétiques ou dans des contextes syntaxiquement libres ;

2) que la structure kāna sa-yaf'alu admet, à côté d'une valeur de futur dans le passé, tout un éventail de valeurs plus ou moins fictives ou contrefactuelles ;

3) que ces valeurs appartiennent toutes au champ sémantico-logique de l'irréalisé et que kāna sa-yaf'alu apparaît comme étant le moyen d'exprimer un fait qui n'a pas eu lieu ;

4) que l'interprétation factuelle de type futur historique n'y est logiquement pas représentée ;

5) que la répartition diatopique des occurrences permet d'affirmer qu'il s'agit d'une structure bien ancrée dans le monde arabe.

Plus généralement, cette brève étude montre à quel point le linguiste se trouve démuni lorsqu'il souhaite décrire un fait syntaxique propre à la langue arabe, n'ayant comme seules références que les occurrences qu'il a bien pu trouver : il ne peut compter ni sur les grammaires arabes, ni sur les grammaires arabisantes, toutes deux résultant d'un processus d'accrétion qui a éloigné la langue décrite de ses descriptions ${ }^{43}$. Surtout, c'est l'absence de grammaire historique de l'arabe qui demeure le plus pesant pour le linguiste : il devient urgent de s'atteler à un tel travail archéologique, en décrivant scrupuleusement à partir de textes authentiques la syntaxe de l'arabe à travers les âges, les lieux et les variétés. Seule une étude de ce type pourrait permettre au linguiste d'envisager avec certitude l'origine d'un phénomène. En faisant le point des descriptions de kāna sa-yaf'alu dans les grammaires d'arabe moderne, on a pu constater une certaine facilité à comprendre tout phénomène rare ou original en arabe comme étant un calque des langues européennes ${ }^{44}$. Or, en l'absence d'une documentation complète sur cette structure, il semble délicat de se prononcer de manière catégorique sur l'origine de l'emploi, même si le lecteur aura compris que l'auteur de ces lignes, à partir d'arguments syntaxiques et logico-sémantiques

\footnotetext{
${ }^{43}$ Sur le concept de sui-langue arabe classique et le phénomène d'accrétion propre aux grammaires arabes, $c f$. Pinon, Catherine, "Les enjeux épistémologiques et didactiques d'une grammaire arabe fondée sur corpus », dans Teddy Arnavielle (éd.), Voyages grammairiens, Paris, L’Harmattan, 2012, p. 83-101.

${ }^{44} \mathrm{Il}$ y a près d'un siècle, Marcel Cohen, dans Le système verbal sémitique et l'expression du temps, Paris, Ernest Leroux, 1924, p. 298, mettait en garde contre l'attitude visant à attribuer à une structure originale une cause extérieure, alors qu'une étude minutieuse de l'évolution du système de la langue peut souvent révéler des origines endogènes : « 11 faut se défier d'explications trop faciles par des influences de substrat ». Il apportait pour cela de nombreux arguments.
} 
notamment, penche dans ce cas précis pour une origine endogène.

Bibliographie

Badawi, el-Sayyid, Carter, Michael et Gully, Adrian, Modern Written Arabic : A comprehensive Grammar, London / New York, Routledge, 2004.

Buckley, Ronald, Modern Literary Arabic: A Reference Grammar, Beyrouth, Librairie du Liban, 2004, nouvelle édition, 2007.

Cohen, Marcel, Le système verbal sémitique et l'expression du temps, Paris, Ernest Leroux, 1924.

Fleisch, Henri, L'Arabe classique : esquisse d'une structure linguistique, Beyrouth, Dar el-Machreq, 1956, édition revue et augmentée, 1968.

Giolfo, Manuela, Les systèmes hypothétiques en 'in de l'arabe classique: étude syntaxique et sémantique : une hypothèse modale, Aix-en-Provence, thèse de doctorat inédite, 2010.

Hassanein, Azza, Modern Standard Arabic Grammar: A concise guide, Le Caire, Dār al-kutub, 2006.

Larcher, Pierre, «Une occurrence ancienne de la structure kāna sa-yaf'alu en arabe écrit », à paraître.

Larcher, Pierre, «Les “complexes de phrases” de l'arabe classique», Kervan, Rivista internazionale di studii afroasiatici / International Journal of Afro-Asiatic Studies, Juillet 2008, $\mathrm{n}^{\circ}$ 6, 2009, p. 29-45.

Larcher, Pierre, «Les systèmes hypothétiques en law de l'arabe classique », BEO LV, Damas, Institut Français d'Études Arabes, 2003, p. 265-285.

Larcher, Pierre, "Y-a-t-il un auxiliaire verbal en arabe ?», dans Jean-Louis Duchet, L'Auxiliaire en question, Travaux linguistiques du CERLICO, 2, Rennes, Presses Universitaires de Rennes, 1990, p. 95-121.

Pinon, Catherine, "Les valeurs de kāna en arabe contemporain», Romano-Arabica XIII, Arab Linguistic, Literary and Cultural Studies, Bucarest, Presses de l'Université de Bucarest, 2013, p. 305-322.

Pinon, Catherine, La nébuleuse de kān: classification des différents emplois de kāna / yakūnu à partir d'un corpus d'arabe contemporain, dir. Pierre Larcher, Aix-en-Provence, thèse de doctorat inédite, 2012.

Pinon, Catherine, «Les enjeux épistémologiques et didactiques d'une grammaire arabe 
fondée sur corpus », dans Teddy Arnavielle (éd.), Voyages grammairiens, Paris, L'Harmattan, 2012, p. 83-101.

Ryding, Karin, A Reference Grammar of Modern Standard Arabic, Cambridge, Presses Universitaires de Cambridge, 2005, 3 ème édition, 2007.

Sartori, Manuel, « Pour une approche relationnelle de la conditionnelle en arabe littéraire moderne », Arabica LVII, 2010, p. 68-98.

Sartori, Manuel, «L'évolution des conditionnelles en arabe égyptien contemporain », BEO 58, 2009, p. 233-257.

Schulz, Eckehard, Krahl, Günter et Reuschel, Wolfgang, Standard Arabic: An elementaryintermediate course, Cambridge, Presses Universitaires de Cambridge, 1996, $9^{\text {ème }}$ réédition, 2008.

Sībawayhi, Al-Kitāab, éd. Hārūn 'Abd as-Salām, Le Caire, Al-hay’a l-mișriyya l- 'āmma li-l-kitāb, 5 volumes, 1977.

Versteegh, Kees, « Two conceptions of irreality in Arabic grammar : Ibn Hisâm and Ibn alHâgib on the particle law », dans Pierre Larcher, De la grammaire de l'arabe aux grammaires des arabes, BEO XLIII, Damas, IFEAD, 1991, p. 77-91. 